\title{
A matter of context
}

\author{
Adaptive behaviors in hematopoietic cells can promote homeostasis and enhance immune responses to pathogens, \\ but they can also perpetuate the chronicity of inflammatory or metabolic disorders.
}

mmune cells exert their functions in a variety of tissues and pathophysiological situations. The ability to acquire contextdependent behaviors is best studied in macrophages, which establish residency in a variety of tissues, and in T cells, which can adapt their effector responses to changing environments. However, the ability to adapt to local signals and conditions seems to be a general feature of all immune cells and hematopoietic progenitor cells and reflects their ability to integrate multiple inputs and engage in context-specific transcriptional and metabolic programs. In the current Focus on Adaptation (https:// www.nature.com/collections/adaptation), Nature Immunology presents three specially commissioned Reviews that discuss the mechanisms that allow immune cells and hematopoietic progenitor cells to adapt to diverse physiological conditions and environments, as well as how such adaptation can be advantageous or can perpetuate the chronicity of various disorders.

A range of adaptive behaviors referred to as 'plasticity', 'training,' 'exhaustion' or 'tolerance' have been observed in innate and adaptive immune cells. Natoli and Ostuni review and analyze mechanisms of adaptation and memory in immune cells, with the aim of providing concepts for understanding these processes. The authors point out that in contrast to memory, in which changes in DNA methylation are transmitted to daughter cells and can persist for decades, adaptation is in principle reversible and dynamic - it can be resolved when environmental conditions return to the initial state or can be modified in response to subsequent microenvironmental changes, and it is linked to molecular alterations that can be rapidly erased, even in non-dividing cells. However, some adaptive phenotypes, such as T cell exhaustion, can be remarkably stable and may involve DNA methylation. In addition, adaptive responses in proliferating cells, such as hematopoietic progenitor cells, may be different from those established in non-proliferating cells, as DNA demethylation during replication may allow the propagation of long-lasting epigenetic changes. Ontogeny may also affect the way cells interact with the environment, as cells of different origins might establish different regulatory networks that will respond differently to stimulation.

Fine-tuning of cellular metabolism to changes in the availability of nutrients, oxygen or growth factors allows cells to retain function in various tissues and conditions. In their Review, Pearce and colleagues discuss aspects of the metabolic adaptation of immune cells to tissue residency and function. Genes encoding metabolic-pathway proteins are strongly represented in the tissue-specific signatures of immune cells, which indicates that metabolic flexibility is essential for immune cell function in various microenvironmental niches. One common feature of tissueresident immune cells seems to be the ability to utilize locally available fuels, such as the use of acetate by peritoneal macrophages, the use of lung surfactant by alveolar macrophages and the use of free fatty acids by skin-resident memory $\mathrm{CD}^{+} \mathrm{T}$ cells. The other feature of resident immune cells is the ability to use such fuels to maintain mitochondrial oxidative phosphorylation, a characteristic of cells with long-term viability. The expression of genes encoding molecules involved in lipid metabolism, largely under the control of the transcription factor PPAR $\gamma$, seems to be a requirement for immune cell residence in non-lymphoid tissues, as documented for macrophages, regulatory $\mathrm{T}$ cells and memory $\mathrm{T}$ cells. On the other hand, inflammatory activation requires aerobic glycolysis. As the authors point out, this allows macrophages to remain viable in inflamed, hypoxic environments and thus might also represent an adaptation mechanism.

Hematopoietic stem and progenitor cells (HSPCs) in the bone marrow sense peripheral inflammation or infection and adapt to it, mostly though increased proliferation and differentiation toward the myeloid lineage. In their Review, Chavakis and colleagues point out that HSPCs are more sensitive to exogenous stimuli than traditionally thought, owing to their expression of receptors for microbial products and for inflammatory cytokines. The underlying mechanisms and the functional outcomes of HSPC adaptation to inflammatory stimuli have only begun to be understood. Although the adaptation of progenitor cells in response to infection can be beneficial to the outcome of infection, it may also cause the exhaustion of HSPCs, which is mediated by DNA damage associated with replication stress and leads to compromised self-renewal. HSPC adaptation may also contribute to the chronicity of inflammatory or metabolic pathologies such as obesity, diabetes or atherosclerosis, in which inflammation and inflammatory adaptations of hematopoietic progenitor cells establish reciprocally reinforced interactions. Adaptation mechanisms in progenitor cells are also the underlying mechanism of trained immunity.

Adaptation, and persistence of the adapted state, can be either advantageous or detrimental. In autoimmunity, T cell exhaustion can be protective. However, $\mathrm{T}$ cell exhaustion has negative consequences in cancer, through the failure to control tumors. Trained immunity may enhance protection against infectious agents but may also exacerbate the chronicity of metabolic diseases. The concept might extend to nonimmune cells as well, as adaptation in skin epithelial stem cells or lung basal cells can enhance tissue regeneration at barrier sites or drive tissue dysfunction during allergy. Understanding the mechanisms of cell adaptation and the context in which this occurs might prove essential for the rational and effective design of therapeutic approaches.

Published online: 18 June 2019 https://doi.org/10.1038/s41590-019-0432-z 